\title{
Does COVID-19 alter the oxyhemoglobin dissociation curve? - An observational cohort study using a mixed-effect modelling
}

https://doi.org/10.1515/cclm-2021-0387

Received March 30, 2021; accepted June 9, 2021;

published online June 21, 2021

Keywords: COVID-19; hemoglobin; hypoxemia; oxygen saturation; oxyhemoglobin dissociation curve.

To the Editor,

Mechanisms leading to hypoxemia in severe acute respiratory syndrome coronavirus-2 (SARS-CoV2) infection are multifactorial and not fully understood [1]. Coronavirus disease-2019 (COVID-19) is characterized by atypical acute respiratory distress syndrome (ARDS) with severe hypoxemia despite initially preserved lung compliance, usually reduced in typical ARDS [2]. Potential mechanisms have been investigated including diffuse pulmonary microembolism [3], deregulated hypoxemic reflex vasodilatation [4], and altered hemoglobin-mediated oxygen transport [5]. In silico models developed in a non-peer-reviewed work predicted that SARS-CoV2 would exhibit surface proteins able to bind to hemoglobin and alter its affinity to oxygen [5].

*Corresponding author: Bruno Mégarbane, Réanimation Médicale et Toxicologique, Hôpital Lariboisière, Fédération de Toxicologie, APHP, INSERM UMRS-1144, Université de Paris, 2 Rue Ambroise Paré, 75010, Paris, France, E-mail: bruno.megarbane@lrb.aphp.fr. https://orcid. org/0000-0002-2522-2764

Mikael Laredo, Réanimation Médicale et Toxicologique, Hôpital Lariboisière, Fédération de Toxicologie, APHP, INSERM UMRS-1144, Université de Paris, Paris, France; and Institut de Cardiologie, Groupe Hospitalier Pitié-Salpêtrière, APHP, Sorbonne Université, Paris, France

Emmanuel Curis, EA 7537 BioSTM, faculté de pharmacie de Paris \& SBIM, Hôpital Saint-Louis, APHP, Université de Paris, Paris, France Elisabeth Masson-Fron, Département de Biochimie, Hôpital Lariboisière, APHP, Université de Paris, Paris, France

Sebastian Voicu, Réanimation Médicale et Toxicologique, Hôpital Lariboisière, Fédération de Toxicologie, APHP, INSERM UMRS-1144, Université de Paris, Paris, France
However, such interaction has not been demonstrated experimentally or clinically. No significant differences in hemoglobin affinity for oxygen were found when comparing oxyhemoglobin dissociation curves in 14 COVID-19 patients to those in 11 healthy volunteers [6]. Yet, the limited sample size requiring data extrapolation in the low oxygen saturation $\left(\mathrm{SO}_{2}\right)$ area of the partial oxygen pressure $\left(\mathrm{PO}_{2}\right)$ curve, the extremely different physiological conditions in the two groups and the exclusion of $\mathrm{PCO}_{2}$ and $\mathrm{pH}$ from the $\mathrm{PO}_{2} / \mathrm{SO}_{2}$ curve modeling prevented definitive conclusions. Here, we assessed the impact of COVID-19 on hemoglobin affinity to oxygen by modeling the $\mathrm{PO}_{2} / \mathrm{SO}_{2}$ relationships in COVID-19 and non-COVID-19 ARDS patients.

We collected blood gas analyses (ABL90 Flex, Radiometer Medical ApS, Denmark; operating temperature, $37^{\circ} \mathrm{C}$ ) in all consecutive COVID-19 and non-COVID-19 ARDS patients admitted to our intensive care unit in MarchDecember 2020 and January 2018-December 2019, respectively. COVID-19 was diagnosed using the standard RT-PCR technique in swabs performed in upper respiratory airways with Cobas-SARS-CoV-2 kits ${ }^{\circledR}$ (Roche, France; sensitivity limit, 40 cycles). $\mathrm{SO}_{2}$ data were fitted using the sigmoidal Hill model: $\mathrm{SO}_{2}=\frac{\mathrm{PO}_{2}^{y}}{\left(\mathrm{P}_{50,0}+\alpha\left(\mathrm{PCO}_{2}-38\right)+\beta(\mathrm{pH}-7.4)^{\gamma}+\mathrm{PO}_{2}^{y}\right.}+\varepsilon$, where $\varepsilon$ is a random variable assumed Gaussian with mean zero and variance $\sigma^{2} ; \mathrm{P}_{50,0}$, the $\mathrm{PO}_{2}$ value when hemoglobin is $50 \%$-saturated with oxygen $\left(\mathrm{P}_{50}\right)$ at $\mathrm{PCO}_{2}=38 \mathrm{mmHg}$ and $\mathrm{pH}=7.40 ; \alpha$ and $\beta$, the parameters quantifying $\mathrm{PCO}_{2}$ - and pH-related effects on $\mathrm{P}_{50}$, respectively. The four model parameters $\left(P_{50,0}, \alpha, \beta\right.$, and $\left.\gamma\right)$ were assumed different in each patient, using a random effect approach. The parameter $\theta$ was modeled as a Gaussian random variable of mean $\mu_{\theta}$ and variance $\sigma_{\theta}^{2}$. All random variables were assumed independent of each other, between patients and of $\varepsilon$. To allow for any effect of COVID-19, the mean value for each parameter was modeled as $\mu_{\theta}=\mu_{\theta \text {,ref }}+\delta_{\theta} \cdot \mathbf{1}_{\text {COVID }}$, where $\mathbf{1}_{\text {COVID }}=1$ for COVID-19 and $=0$ for non-COVID-19 patients. In each group, the "average patient" was defined as the patient with the median values for each $\mathrm{P}_{50,0}, \alpha, \beta$, and $y$ parameter. 
This approach is similar to mixed-effect models used in population pharmacokinetics [7]. COVID-19-related effects on the oxyhemoglobin dissociation were considered significant if the $95 \%$-confidence interval $(95 \% \mathrm{CI})$ of at least one of $\delta \mathrm{P}_{50,0}, \delta \alpha, \delta \beta$, or $\delta y$ did not include zero. The model was fitted in $\mathrm{R}$ version-3.6.3 [8] using the nlme function (nlme package, version-3.1.151) [9] based on the maximum likelihood method. The $95 \%$ CIs were determined using the Wald method. Model assumptions were checked graphically using standard validation methods including visual predictive checks and normal distribution checks by quantile-quantile plots. The results are expressed as median (25th-75th percentiles) or percentages. Comparisons were performed using Mann-Whitney and Chi-square tests as appropriate.

Overall, 5,291 and 3,449 blood gas measurements obtained from 124 COVID-19 (age, 59 years [50-71]; M/F sex ratio, 2.54) and 144 non-COVID-19 patients (age, 61 years [49-73]; M/F sex ratio, 2.87), respectively, were included ( $\mathrm{p}=0.41$ for age, $\mathrm{p}=0.77$ for gender). ARDS etiologies in non-COVID-19 patients included bacterial pneumonia $(\mathrm{n}=80,56 \%)$, influenza $(\mathrm{n}=32,22 \%)$, and non-infectious injuries $(n=44,31 \%)$. Invasive mechanical ventilation was used in $74(60 \%)$ vs. 76 patients $(53 \%)(p=0.34)$ and extracorporeal membrane oxygenation (ECMO) in six (5\%) vs. eight patients $(6 \%)(p=0.99)$. The number of blood gas measurements per patient was 18 [4-75] and 13 [4-26] in the COVID-19 and non-COVID-19 patients, respectively $(\mathrm{p}=0.01)$. Average hemoglobin was $11.1 \mathrm{~g} / \mathrm{dL}$ [9.6-13.2] vs. $10.8 \mathrm{~g} / \mathrm{dL}$ [9.4-12.7] $(\mathrm{p}=0.4)$. Average $\mathrm{SO}_{2}$ was $95.7 \%$ [93.9-96.8] vs. 95.1\% [91.7-96.3] $(\mathrm{p}=0.03)$, average $\mathrm{PO}_{2}$ $107 \mathrm{mmHg}$ [91-128] vs. $95 \mathrm{mmHg}$ [77-115] $(\mathrm{p}=0.002)$, average $\mathrm{PCO}_{2} 43 \mathrm{mmHg}$ [38-48] vs. $42 \mathrm{mmHg}$ [37-49] $(\mathrm{p}=0.87)$ and average $\mathrm{pH} 7.41[7.38-7.45]$ vs. 7.40 [7.34-7.44] ( $\mathrm{p}=0.04)$.

Our model well fitted the observed data showing, as expected, that $\mathrm{PCO}_{2}$ increases $\mathrm{P}_{50}$ ( $\alpha$ estimate $>0,95 \% \mathrm{CI}$ excluding zero) and $\mathrm{pH}$ decreases $\mathrm{P}_{50}$ ( $\beta$ estimate $<0,95 \%$ $\mathrm{CI}$ excluding zero; Figure 1). The model-calculated $\mathrm{P}_{50,0}$, $\alpha, \beta$, and $\gamma$ for each patient are given in Figure 2. $P_{50,0}, \beta$, and $y$ did not significantly differ between COVID-19 and non-COVID-19 patients $\left(95 \% \mathrm{CI}\right.$ of $\delta \mathrm{P}_{50,0}, \delta y$, and $\delta \beta$ all included zero) whereas $\alpha$ did $(\delta \alpha=-0.025,95 \% \mathrm{CI}$ $\left.\left[-0.051 ;-1.7 \times 10^{-4}\right]\right)$. This decrease in $\alpha$, representing the

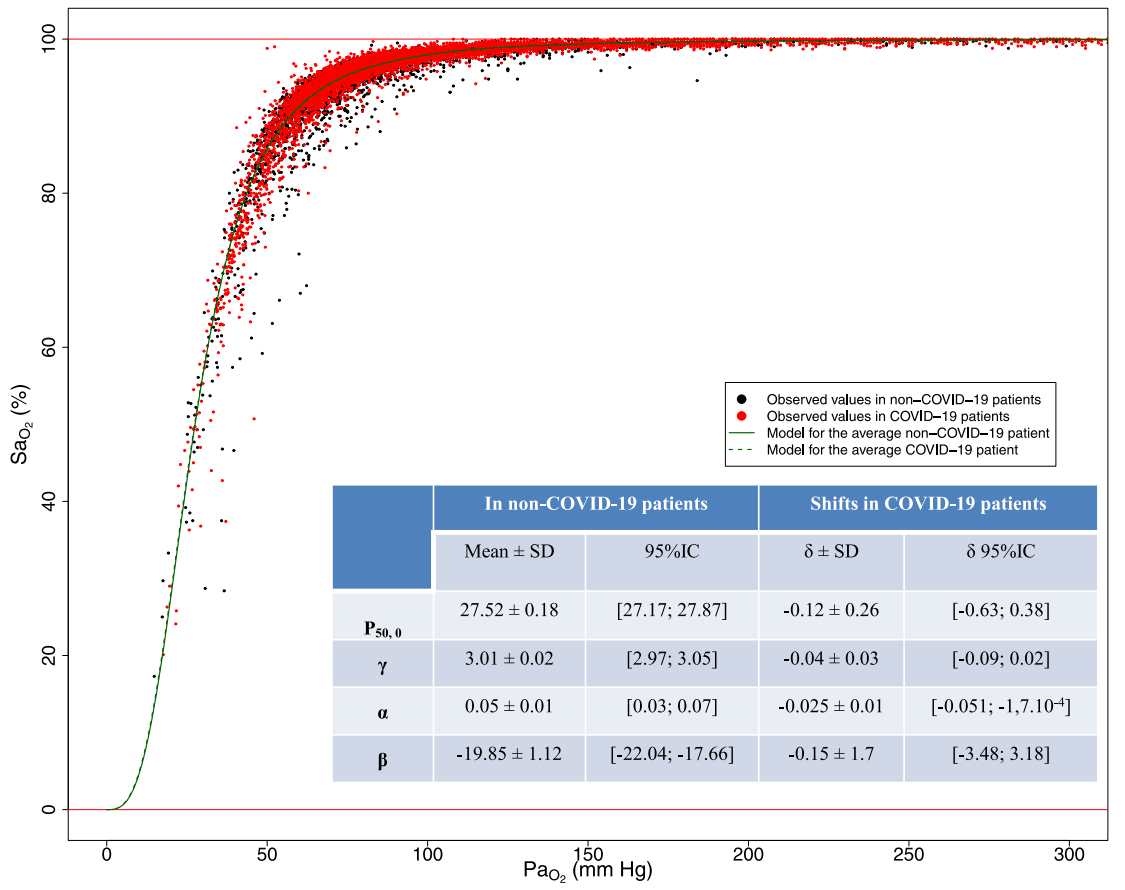

Figure 1: The oxyhemoglobin dissociation curve in COVID-19 and non-COVID-19 patients.

Single measurements are displayed as dots (red, 5,291 measurements in 124 COVID-19 patients; black, 3,449 measurements in 144 non-COVID-19 patients). $\mathrm{SO}_{2}$ data were fitted using the sigmoidal Hill model: $\mathrm{SO}_{2}=\frac{\mathrm{PO}_{2}^{\gamma}}{\left(\mathrm{P}_{50,0}+\alpha\left(\mathrm{PCO}_{2}-38\right)+\beta\left(\mathrm{pH}^{2} .4\right)\right)^{\gamma}+\mathrm{PO}_{2}^{\gamma}}+\varepsilon$, where $\mathrm{P}_{50,0}$, the $\mathrm{PO}_{2}$ value when hemoglobin is $50 \%$-saturated with oxygen $\left(\mathrm{P}_{50}\right)$ at $\mathrm{PCO}_{2}=38 \mathrm{mmHg}$ and $\mathrm{pH}=7.40 ; \alpha$ and $\beta$, the parameters quantifying $\mathrm{PCO}_{2}$ - and $\mathrm{pH}$-related effects on $\mathrm{P}_{50}$, respectively; and $\varepsilon$ is a random variable assumed Gaussian with mean zero and variance $\sigma^{2}$. Inter-patients variability was estimated at $1.5,0.16,0.06$, and $9.79 \mathrm{mmHg}$ for $\mathrm{P}_{50}, 0, \gamma, \alpha$, and $\beta$, respectively (standard deviation of the random effect). The theoretical $\mathrm{PO}_{2} / \mathrm{SO}_{2}$ curves for the average patient in each group (continuous green line, COVID-19 patients; dashed green line, non-COVID-19 patients) are superimposable. The $95 \%$-confidence intervals of the difference $(\delta)$ between COVID-19 and non-COVID-19 patients regarding $P_{50,0}, \beta$, and $\gamma$ but not $\alpha$ included 0 . 

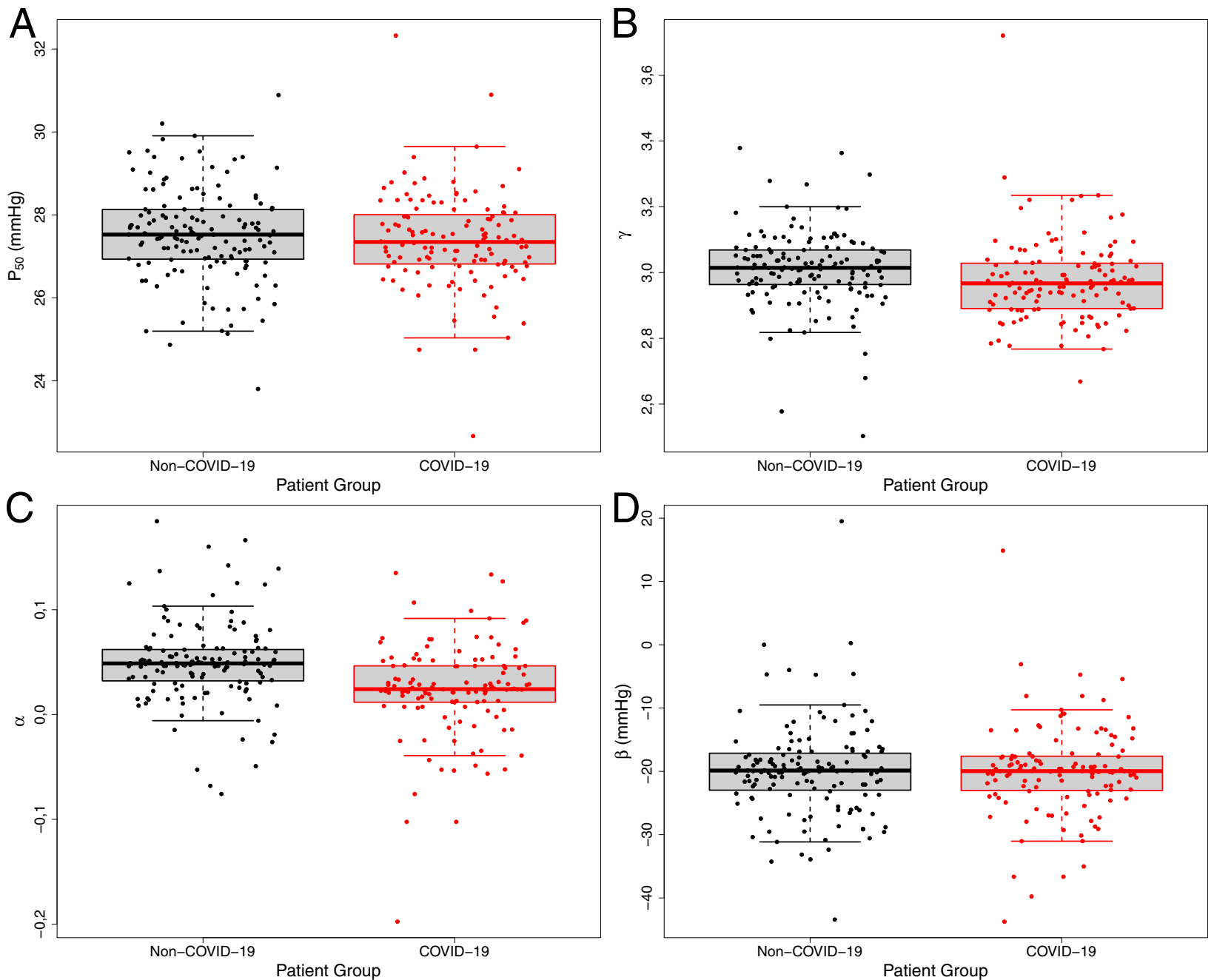

Figure 2: Individual parameters of the oxyhemoglobin dissociation curve in COVID-19 and non-COVID-19 patients.

Values of $P_{50}(A), \gamma$ (Hill coefficient), (B), $\alpha$ (parameter quantifying $\mathrm{PCO}_{2}$-related effects on $\mathrm{P}_{50}$ ), (C), and $\beta$ (parameter quantifying pH-related effects on $P_{50}$ ), (D) are the fit result, obtained as the sum of the average value, corrected for COVID-19 when needed, and the individual random effect value. Hence, these values cannot be used for statistical inference; only the confidence interval of the model parameters can be interpreted.

slope of the effect of $\mathrm{PCO}_{2}$ on $\mathrm{P}_{50}$, from 0.049 to 0.025 in COVID-19 patients suggested a decreased sensitivity of $\mathrm{P}_{50}$ to $\mathrm{PCO}_{2}$. However, the $95 \% \mathrm{CI}$ was large and its upper bound almost zero. Otherwise, when including mechanical ventilation and ECMO as covariates, decrease of $\mathrm{P}_{50}$ sensitivity to $\mathrm{PCO}_{2}$ in COVID-19 patients was still present $(\delta \alpha=-0.06914,95 \% \mathrm{CI}[-0.102 ;-0.036]$ in nonECMO-treated and non-mechanically ventilated patients). The theoretical $\mathrm{PO}_{2} / \mathrm{SO}_{2}$ curves for an average patient $\left(\mathrm{PCO}_{2}=38 \mathrm{mmHg}, \mathrm{pH}=7.4\right)$ in each group were superimposable.

Although applied for the first time to characterize the oxyhemoglobin dissociation curve, our mixed-effect model including $\mathrm{pH}$ and $\mathrm{PCO}_{2}$ as covariates showed only a slightly significant SARS-CoV2-induced effect on the
$\mathrm{PO}_{2} / \mathrm{SO}_{2}$ relationships that appeared less sensitive to $\mathrm{PCO}_{2}$ in COVID-19 patients. Similar results were found if limiting the analysis to blood gas samples during the time of positive RT-PCR. However, since the Wald method is known to give too narrow $95 \% \mathrm{CIs}$ and correction for multiplicity resulted here in non-significant comparisons, this finding should be considered cautiously. In any case, the clinical relevance of such SARS-CoV2-related effects seems limited. Interestingly, a significant $\sim 0.8 \mathrm{mmHg}$ decrease in $\mathrm{P}_{50}$ once corrected for $\mathrm{pH}$ and $\mathrm{PCO}_{2}$ was similarly reported in COVID-19 patients, with persistent left shift when only one value per patient was considered $(n=43)$ [10]. Therefore, an overcorrection for $\mathrm{PCO}_{2}$ when using the classical formula in COVID-19 patients may be hypothesized, artificially decreasing the apparent $\mathrm{P}_{50}$. 
Only samples encompassing extreme $\mathrm{PO}_{2}$ and $\mathrm{SO}_{2}$, as provided in our study, allow assessment of the model accuracy in the far-left part of the $\mathrm{PO}_{2} / \mathrm{SO}_{2}$ curve. Additionally, we compared two groups with similar physiological settings and our model included the effects of $\mathrm{pH}$ and $\mathrm{PCO}_{2}$. Finally, our findings were consistent with another work showing that hemoglobin-oxygen dissociation curves generated from 11 critically ill COVID-19 patients matched the idealized curve, similarly to eleven non-COVID-19 patients [11].

Our study has limitations. Our mixed-effect model was used without prior validation in an external population. However, given its proximity with the Hill model and based on good agreement as shown in Figure 1, our model appeared adequate to describe the $\mathrm{PO}_{2} / \mathrm{SO}_{2}$ relationships while allowing potential patient-related effects. We could not rule out the presence of COVID-19 patient subpopulations in which hemoglobin physiology is affected. Moreover, our ex vivo modeling of oxygen affinity to hemoglobin may not take into account the presence of in vivo modulators specifically associated with COVID-19, even though no evidence suggests it to date.

To conclude, our study provides no evidence for clinically relevant SARS-CoV2-related alterations in the oxyhemoglobin dissociation curve. However, despite limited expected clinical consequences, we found a decrease impact of $\mathrm{PCO}_{2}$ on $\mathrm{P}_{50}$ in the COVID-19 patients.

Research funding: None declared.

Author contributions: M.L. and B.M. were responsible for study design. M.L., E.M.F., S.V. and B.M. were responsible for data collection and analysis. E.C. was responsible for the modeling and statistical analysis. M.L., E.C. and B.M. were responsible for first draft writing and manuscript finalization. All authors have accepted responsibility for the entire content of this manuscript and approved its submission.

Competing interests: Authors state no conflict of interest. Informed consent: The Ethics Committee waived informed consent from individuals included in this study as no additional intervention related to the research was performed.

Ethical approval: Research involving human subjects complied with all relevant national regulations, institutional policies and is in accordance with the tenets of the Helsinki Declaration (as revised in 2013). The study was part of the COVID-ICU and French COVID-19 cohort registries and approved by our institutional ethics committee (IDRCB, 2020-A00256-33; CPP, 11-2020.02.04.68737).

\section{References}

1. Wiersinga WJ, Rhodes A, Cheng AC, Peacock SJ, Prescott HC. Pathophysiology, transmission, diagnosis, and treatment of coronavirus disease 2019 (COVID-19): a review. J Am Med Assoc 2020;324:782-93.

2. Gattinoni L, Chiumello D, Caironi P, Busana M, Romitti F, Brazzi L, et al. COVID-19 pneumonia: different respiratory treatments for different phenotypes? Intensive Care Med 2020;46:1099-102.

3. Ackermann M, Verleden SE, Kuehnel M, Haverich A, Welte T, Laenger F, et al. Pulmonary vascular endothelialitis, thrombosis, and angiogenesis in Covid-19. N Engl J Med 2020;383:120-8.

4. Reynolds AS, Lee AG, Renz J, DeSantis K, Liang J, Powell CA, et al. Pulmonary vascular dilatation detected by automated transcranial Doppler in COVID-19 pneumonia. Am J Respir Crit Care Med 2020;202:1037-9.

5. Liu W, Li H. COVID-19: attacks the 1-beta chain of hemoglobin and captures the porphyrin to inhibit human heme metabolism. Available from: https://chemrxiv.org/articles/preprint/ COVID-19_Disease_ORF8_and_Surface_Glycoprotein_Inhibit_ Heme_Metabolism_by_Binding_to_Porphyrin/11938173 [Accessed 30 March 2021].

6. Daniel Y, Hunt BJ, Retter A, Henderson K, Wilson S, Sharpe CC, et al. Haemoglobin oxygen affinity in patients with severe COVID19 infection. Br J Haematol 2020;190:e126-7.

7. Dartois C, Brendel K, Comets E, Laffont CM, Laveille C, Tranchand $B$, et al. Overview of model-building strategies in population $\mathrm{PK} /$ PD analyses: 2002-2004 literature survey. Br J Clin Pharmacol 2007;64:603-12.

8. The R Core Team. R: a language and environment for statistical computing. Vienna, Austria: R Foundation for Statistical Computing; 2020.

9. Pinheiro J, Bates D, The R Core Team. nlme: linear and non-linear mixed effects model. Available from: https://svn.r-project.org/ R-packages/trunk/nlme/ [Accessed 30 March 2021].

10. Vogel DJ, Formenti F, Retter AJ, Vasques F, Camporota L. A left shift in the oxyhaemoglobin dissociation curve in patients with severe coronavirus disease 2019 (COVID-19). Br J Haematol 2020;191: 390-3.

11. DeMartino AW, Rose JJ, Amdahl MB, Dent MR, Shah FA, Bain W, et al. No evidence of hemoglobin damage by SARS-CoV-2 infection. Haematologica 2020;105:2769-73. 